\title{
Applying for ethical approval for research in the United Kingdom
}

\section{A Smajdor,}

School of Medicine, Health Policy and Practice, University of East Anglia, Norwich NR4 7TJ Riverside Research Ethics Committee, Charing Cross Hospital, London W6 8RF

\section{R Sydes}

Cancer Group, MRC Clinical Trials Unit, London NW1 2DA

Cambridgeshire 4 Research Ethics Committee, Cambridge CB21 5XB

\section{Gelling}

Faculty of Health and Social Care, Anglia Ruskin University, Cambridge CB1 1PT

Cambridgeshire 4 Research Ethics Committee, Cambridge CB21 5XB

\section{Wilkinson}

School of Medicine, Health Policy and Practice, University of East Anglia, Norwich NR4 7TJ Institute of Health Research Ethics Committee, University of East Anglia

Correspondence to: M Wilkinson mark.wilkinson@nnuh.nhs.uk

This article offers practical advice on how to expedite the ethical review process

\section{Glossary of useful terms}

\section{Chief investigator:}

Person with overall responsibility for the research. All applications for ethical review should be submitted by the chief investigator

\section{Clock:}

Period allowed for the ethical review of a new application or substantial amendment. The clock starts on receipt of a valid application

\section{Employing organisation:}

Organisation employing the chief investigator, other investigators, or research collaborators. Employers remain liable for the work of their employees

Integrated research application system (IRAS):

Online application system used to apply for most permissions and approvals for research in health and social care in the UK (www.myresearchproject.org.uk/)

Local collaborator:

Person undertaking certain types of straightforward research not requiring the appointment of a principal investigator and a site specific assessment (see "site specific exemption"). Local collaborators at NHS sites should still seek approval from the research and development office

\section{Principal investigator:}

Person responsible for the local research site in studies with multiple sites

\section{Protocol:}

Document that describes the objectives, design, methodology, statistical considerations (or other methods of data analysis), and organisation of a research study

\section{Research site:}

Organisation or unit responsible for conducting any of the research procedures in a study at a particular locality

\section{Site specific assessment:}

Assessment of the suitability of the investigator, site, and

facilities of each research site when research is taking place in multiple sites

Site specific assessor:

Body responsible for undertaking a site specific assessment, either the research and development office for an NHS site or an appropriate local research ethics committee for a non-NHS site 


\section{Site specific exemption:}

If only low risk procedures are being undertaken, site specific assessment may not be needed. The application should indicate whether this is thought to be the case (guidance is provided at: http://www.nres.npsa.nhs.uk/EasySiteWeb/GatewayLink.aspx?alld=393 ). The final decision on whether site specific assessment is needed will be made by the research ethics committee

\section{Sponsor:}

Person with ultimate responsibility for the initiation, management, and financing (or arrangement of financing) of a clinical trial

Applying for ethical review in the United Kingdom can be time consuming and burdensome for researchers. ${ }^{1-3}$ The process can often be made easier by implementing relatively minor changes in the early stages of drawing up the application. In this article we discuss the practicalities of preparing an application for ethical review, and the process by which decisions are made. We offer advice on how to avoid common problems when applying to a research ethics committee and provide information on the integrated research application system, launched in January 2008.

\section{What is the function of research ethics committees?}

Ethical review is designed to ensure that the benefits of research do not come at the expense of exploiting the vulnerability of research participants. ${ }^{4}$ Germany led the way in drafting an ethicolegal framework for research in 1900, after it emerged that patients had been infected with syphilis by a doctor who was trying to develop a vaccine for the disease. ${ }^{56}$ Further guidelines issued in 1931 by the German Reich minister of the interior emphasised patient autonomy and informed consent.

Nazi doctors shortly afterwards embarked on a series of experiments that appalled the world. When full details of the Nazis' research programme were revealed during the Nuremberg trials, it became clear that existing frameworks were insufficient. Accordingly, the Nuremberg Code was drafted in 1947, followed by a number of national and international guidelines, codes of conduct, and declarations. The Declaration of Helsinki first published by the World Medical Association in 1964, and most recently updated in 2008, is perhaps the most influential of these. Yet these codes have not prevented unethical research, examples of which still emerge today. ${ }^{7-9}$

\section{The UK's research ethics process}

The United Kingdom has tackled these problems by requiring that research on human subjects must be reviewed by independent ethics committees. Seeking ethical review is now a well established part of the research process. But what is it that research ethics committees actually look for in an application? Box 1 lists some of the key questions that committees consider

\section{Before starting the process of application}

Designing a study with a view to mitigating or minimising ethical problems from the outset is half the battle. The next step is to draft the participant information sheet and consent forms (box 2). Many research ethics committee members start their review by reading the participant information sheet for a concise understanding of the purpose of the research and what it will entail. If the information provided does not convey this understanding, it will cause delays in obtaining approval, however good the rest of the application may be. 
Participant information sheets may vary with the nature of the research. Projects involving children need comprehensible and age appropriate information. In contrast, some participants with long term conditions may be familiar with medical terminology relating to their condition. But as a general rule, clear simple language should be used (around $16 \%$ of adults in England have literacy levels at or below those expected of an 11 year old). ${ }^{10}{ }^{11}$ Patients and representative groups are often happy to review patient information sheets and other documents. Using a standard structure can ensure that all the necessary material is provided in a logical format. The National Research Ethics Service publishes guidance on this subject (www.nres.npsa.nhs.uk/EasySiteWeb/GatewayLink.aspx?alld=338).

Once the study has been designed and the participant information sheets drawn up, the process of seeking ethical review can begin.

\section{Box 1 | What do research ethics committee members consider in an application?}

The scientific and ethical importance of the study

Is it likely to provide useful new information? Is it investigating an area in which the need for further research is compelling?

The likelihood that the study will achieve its aims

Is the design of the study adequate to fulfil what it sets out to establish?

Risks involved

Are patients likely to be harmed physically or psychologically as a result of participating? Are the potential harms proportionate to the likely benefits of the study? Have all possible means of reducing risk been explored?

Use of vulnerable groups

Does the study involve children, prisoners, or those who lack the capacity to make informed decisions? If so, is their inclusion justified?

Methods of recruitment

How are potential participants being identified? Is there a risk of coercion?

Consent procedure

Are adequate procedures in place for gaining consent?

Patient information sheet

Is clear, comprehensible, and honest information provided?

Confidentiality and protection of data

Who will be accessing records or data? Will it be anonymised? Where will it be kept?

Feedback to participants

Participants have a stake in the research. What arrangements have been made for reporting findings back to them? (Participants are unlikely to have access to medical journals in which studies are published, and such studies are unlikely to be readily understood by them.)

Destruction of samples or data

How long will tissue samples or patient data be kept? Will it be used in future research? 


\section{Box 2 General pointers for writing participant information sheets}

- Keep it as short and simple as possible while retaining the relevant information-avoid information overload

- Avoid complex or specialised terminology

- Number the points-such a structure helps participants to focus and helps the ethics committee to draw out clarifications

- Include a clear summary of risks, detailing both the expected frequency and severity of Complications

- Include information about the risk to unborn children if relevant

- If the patient information sheet suggests that the participants seek advice from a "doctor" it should be clear whether - this means their general practitioner, a consultant, or the research doctor

- Information sheets aimed at child participants must be suitable for the appropriate age group; younger children need $\cdot$ simple language and pictorial representation of ideas, whereas teenagers may feel "talked down to" if information is oversimplified

- In principle, excluding participants who do not speak English is unethical, but it may be justified when questionnaires • have been validated in English only. For larger studies research ethics committees will expect formal, professional, and validated translation of participant information sheets. Family members are not usually allowed to be translators

- The committee will want to know how study findings will be reported to participants. Publication in medical journals • that are not accessible by most participants will not Suffice

\section{What is the process for research ethics application in the UK?}

\section{Create an account on the online integrated research application system}

In the past, separate applications had to be submitted for ethics and for research governance approval. (Research governance is the province of research and development departments, and it is designed to ensure transparency and high scientific standards. More information can be found on the Department of Health website. $)^{12}$ Researchers can now save unnecessary duplication by using one central system to enter information about their project, and complete the forms for review by all regulatory and governance bodies www.myresearchproject.org.uk/

Several different approvals or applications may be generated by the system, depending on the type of research. Answers to the first questions determine which forms will be generated, so mistakes made at this stage may result in invalid or incomplete applications. This article will focus on the ethics form. Some fields will be autofilled using data entered at the outset. Problems can arise if information in the ethics application contradicts the protocol, so cross checking for consistency is advisable. It is important to use lay language in the free text responses describing research questions, objectives, scientific justification, and methods. Research ethics committees always include lay members, and even specialists may struggle with complex terminology.

If researchers bear in mind such ethical considerations when filling in the ethics form, they may greatly speed up the ethical review process. For example, recruiting junior colleagues as research participants poses an ethical problem because these people may feel that refusal could negatively affect their career. If the researchers recognise this problem in advance they can consider whether there is a clear rationale for this approach. If not, the study design can be amended. If there are good reasons for adopting this recruitment strategy, they can be detailed in the application form. 


\begin{tabular}{|l|}
\hline Box 3 | Documentation to be sent to the research ethics office \\
\hline Covering letter \\
\hline Research ethics committee* \\
\hline Site specific information form \\
\hline Research protocol \\
\hline Investigator's brochure or summary of product characteristics $^{*}$ Investigator's curriculum vitae* $^{\star}$ \\
\hline Participant information sheet \\
\hline Participant consent form \\
\hline Letter of invitation to participant \\
\hline Information sheets or letters for general practitioners or consultants \\
\hline Evidence of insurance or indemnity arrangements* \\
\hline Letter from sponsor \\
\hline Letter from statistician \\
\hline Referees' reports \\
\hline Lay synopsis or flowchart of protocol \\
\hline Details of any data monitoring committee \\
\hline Sample diary card or patient card \\
\hline Questionnaire \\
\hline Interview schedules \\
\hline Copies of recruitment advertisements, such as posters \\
\hline
\end{tabular}

\section{Booking a slot with an ethics committee}

When applicants have entered all the relevant project information on the online system, a slot can be booked with a research ethics committee. The type of research being undertaken will determine which ethics committee is appropriate

http://www.nres.npsa.nhs.uk/applicants/apply/applying-for-ethical-review/ Once an ethics committee has been identified, the coordinator of that committee will help with queries related to the process.

\section{Adding the reference number}

After the application has been scheduled for review with a committee, it will be assigned an identifying research ethics committee reference number, which the applicants then add to the online form.

\section{Site specific information}

Site specific assessment may be needed if the research is being undertaken at multiple sites. Site specific information forms can be obtained and filled in via the central system. They should be completed after the research ethics committee has validated the study for ethical review and a reference number has been issued. Site specific assessments from local research ethics committees do not involve re-review of the protocol, they simply assess aspects of the research particular to a specific site-for example, ensuring that adequate facilities are available to conduct the research and the local investigator(s) are appropriately qualified. Further information and guidance on site specific assessment are available at the integrated research application system website. 
An ink signature is required from the chief investigator. However, the system allows for electronic authorisation from the other collaborators. When the forms are ready to be signed and submitted by the applicant, they can be saved in PDF format and printed out. A hard copy of the application form should be sent to the research ethics committee office, together with the submission checklist and all relevant supporting documentation. Supporting documentation will vary depending on the type of research. Box 3 lists the information that may be needed.

The completed paperwork must be received by the ethics committee within four days of the slot being booked. If the paperwork for a study is received less than two weeks before the date of the next meeting, the study will not be reviewed until the subsequent meeting. However, by liaising closely with the committee's coordinator, researchers can find out the dates of forthcoming meetings and organise themselves accordingly.

\section{Box 4 | What next?}

\section{Approval}

The study can go ahead with no further amendments.

Conditional approval

The ethics committee provides a list of minor amendments to be implemented by the applicants before the study begins. However, the amendments do not have to be re-reviewed by the committee.

\section{Provisional approval}

The decision letter will detail the changes that are needed before approval can be granted. The 60 day clock is suspended until the researcher responds to the points made by the committee. The study cannot start until the committee has approved the changes.

Researchers should respond by sending a letter that explains what action has been taken on each point. Copies of all modified documents with changes tracked and version numbers updated should also be sent.

When the response is received, a subcommittee will review the changes. Ongoing problems related to the modifications can be raised, but the committee cannot raise new concerns at this time.

\section{Rejection}

The committee thinks that the study as it stands is not ethically sound. A list of reasons will be given for this decision.

On receiving a rejection, researchers may appeal to another research ethics committee, who will receive the original paperwork. When reviewing such an application, committee members are not bound by the preceding opinion, and it is not uncommon for opinions to be reversed. If a rejection is upheld, advice may be given about how the project could be redesigned.

\section{What happens after submission?}

An ethical opinion must be given within 60 days of submitting the paperwork (the "60 day clock"). Committee meetings are held monthly, and they usually last around three hours. Applications to be considered (around six each meeting) are sent to members two weeks in advance to ensure they have enough time to go through them. Applicants will be informed of the date and location of the meeting, and invited to attend. 
The order in which papers are reviewed is fixed in advance, so that researchers can be given an estimated time for their application to be reviewed. Every effort is made to keep on track, but it is not always possible to predict how long a review will take, so timings are approximate. It is also not possible to stipulate exactly how long the researcher will spend with the committee. This can be just a few minutes to half an hour, depending on the concerns raised by the committee.

\section{Attending the meeting}

Research ethics committees are composed of professionals (pharmacists, statisticians, nurses, or medical practitioners) and lay members. Many committees nominate two lead reviewers for each study, but every member must read each application. The committee identifies the matters to be raised before inviting the researchers into the meeting room.

The discussion between researchers and committee members will cover points that raise ethical concerns plus any aspects of the application that are confusing, contradictory, or otherwise problematic. Queries can often be resolved at the meeting if the researcher is present, so it is advisable to attend if possible. For student research, it is helpful if the supervisor and the student attend, so that both perspectives can be taken into account. Likewise, for clinical trials, the chief investigator should be accompanied by someone overseeing the day to day running of the trial. After the researchers have left, the committee will reconsider the application in the light of what has been discussed. A final decision will be agreed.

\section{The outcome}

A letter informing researchers of the committee's decision is sent to applicants within 10 working days of the meeting. Four outcomes are possible: approval, conditional approval, provisional approval, and rejection (box 4). In our experience fewer than $10 \%$ of projects are rejected. Around $14 \%$ will be approved with no amendments. ${ }^{5}$ The rest will need to make some changes. In many cases, the problems could have been identified and remedied earlier-for example, spelling and grammatical errors in patient information documents.

\section{Conclusion}

Members of research ethics committees are usually dedicated and concerned people who wish to facilitate high quality ethical research. Mutual respect through better understanding is likely to help the work of both reviewers and committee members and allow ethical review to be a collaborative process that improves the quality and value of research, not just a bureaucratic hurdle.

\section{Thanks to Atul Patel, coordinator of Riverside Research Ethics Committee, for his help and advice.}

Funding: No special funding.

Competing interests: None declared.

Provenance and peer review: Not commissioned; externally peer reviewed.

1. Maskell NA, Jones EL, Davies RJO. Variations in experience in obtaining local ethical approval for participation in a multi-centre study QJM 2003;96:305-7.

2. Hallowell N, Cooke S, Crawford G, Parker M, Lucassen A. Ethics and research governance: the views of researchers, health-care professionals and other stakeholders. Clin Ethics 2008;3:85-90.

3. Hackshaw A, Farrant H, Bulley S, Seckl M, Ledermann J. Setting up non-commercial clinical trials takes too long in the UK: findings from a prospective study. J R Soc Med 2008;101:299-304.

4. Schuklenk U. Protecting the vulnerable: testing times for clinical research ethics. Soc Sci Med 2000;51:969-77. 
5. Vollman J, Winau R. Informed consent in human experimentation before the Nuremberg Code. BMJ 1996;313:1445-50.

6. Grodin M. Historical origins of the Nuremberg Code. In: Annas G, Grodin M, eds. The Nazi doctors and the Nuremberg Code: human rights in human experimentation.

Oxford University Press, 1995:121-48.

7. Pappworth MH. Human guinea pigs. Beacon Press, 1967.

8. Young C, Godlee F. Managing suspected research misconduct. BMJ 2007;334:378-9

9. Dyer C. UK must learn from US's mistakes in tackling research misconduct. BMJ 2007;334:1027.

10. Priestley K, Campbell C, Valentine C, Denison D, Buller N. Are patient consent forms for research protocols easy to read? BMJ 1992;305:1263-4.

11. National Literacy Trust. Frequently asked questions about literacy. How many illiterate adults are there in England? www.literacytrust.org.uk/About/FAQs.html\#illiterate.

Department of Health. Research governance framework for health and social care. 2nd ed. 2005. 